\title{
A Novel Fuzzy Data Association Approach for Visual Multi-object Tracking
}

\author{
Liang-Qun* LI, En-Qun LI, Wen-Ming HE \\ ATR Key Laboratory, Shenzhen University, Shenzhen, Guang Dong ,China \\ Shenzhen Chichen Cloud Net Co., LTD, Shenzhen, Guang Dong ,China \\ Shenzhen Pan Go Microsystems Co.,LTD, ,Shenzhen, Guang Dong ,China \\ e-mail: Iqli@szu.edu.cn,67327301@qq.com,18900058@qq.com
}

\begin{abstract}
Multiple object tracking (MOT) is one of the most important research areas in visual surveillance. However, some practical challenges remain to be overcome for implementing this technology, such as occlusion, missed detection, false detection, and abrupt camera motion. In this paper, to the visual multi-object tracking, a novel fuzzy data association algorithm is proposed. In order to incorporate expert experience into the proposed algorithm, a fuzzy inference system based on knowledge is designed, and the fuzzy membership degrees are used to substitute the association probabilities between the objects and observations. The experiment results on several public data sets show that the proposed algorithm has advantages over other state-of-the-art tracking algorithms in terms of efficiency.
\end{abstract}

\section{Introduction}

The objective of multi-object tracking is to estimate the current states of objects based on previous visual measurements up to the current time in a video sequence, such as positions, size, identification (ID), etc. It is very important for many computer vision tasks with applications such as automated surveillance, traffic safety, vehicle navigation, human computer interaction, and robotics [1], [2] With the development of the detection technology of object, detection-based multi-object tracking methods have been extensively studied [3].

A key problem of the tracking-by-detection approach is that of the data association between visual measurements and multiple objects. In order to solve the data association problems, many data association approaches have been proposed in recent decades. To obtain the global optimal solution or sub-optimal solution, Park et al.[1]proposed a binary integer programming formulation for a data association problem which pursues the minimum cost data associations among target measurements via one-to-one, one-to-m, and m-to-one associations. Milan et al. [4] used gradient descent to find strong local minima of complex nonconvex energy that captures image evidence and various physical constraints for tracking. Leibe et al. [5] proposed to perform coupled multiple-object detection and tracking by applying the minimum description length principle, formulate it as a QBP, and solve it by expectation maximization (EM) method. Benfold and Reid [6] employed MCMC to track multiple heads where motion is exploited to detect false positive (FP) detections. Bae et al.[7] proposed a data association with a track existence probability by incorporating the detections into tracks to solve partial occlusions, and a track management method was used to deal with track initialization, link and termination, which can associate terminated tracks for linking tracks fragmented by long-term occlusions.

In this paper, a novel fuzzy logic data association method for online visual multi-object tracking is proposed. The error and the change of error of motion, shape and appearance models are used to construct the fuzzy input variables and fuzzy inference system. In the fuzzy inference system, the association probabilities between observations and objects are replaced by the fuzzy membership degrees, which can incorporate reasoning based on the fuzzy rule base in the same sense as human reasoning.

The rest of this paper is organized as follows. Section 2 describes the proposed multi-object tracking algorithm. Experiment results that compare the performances of all algorithms are presented in Section 3. Finally, some conclusions are provided in Section 4. 


\section{Proposed Multi-Object Tracking Algorithm}

\subsection{Fuzzy Logic Data Association}

To calculate the fuzzy association probabilities $\mu_{i, j}(k)$ between the objects $\left\{\hat{x}_{k-1}^{i}\right\}_{i=1}^{m}$ and observations $\left\{z_{k}^{j}\right\}_{j=1}^{n}$ (or detection responses), a fuzzy inference system (FIS) is designed based on the affinities of motion and appearance. The FIS contains four basic elements: fuzzifier of input variables, fuzzy knowledge-base, fuzzy inference engine, and defuzzifier.

\subsection{System variables}

Suppose that the predicted state of object $i$ at the $k$ th frame is $\quad X=\left\{\hat{x}_{i}(k)\right\}_{i=1}^{m}$,where $\quad \hat{x}_{i}(k)=\left(x_{i}(k), v_{x_{i}}(k), y_{i}(k), v_{y_{i}}(k)\right)$. $x_{i}(k)$ denotes the $\mathrm{x}$-coordinate of the object's prediction position, $y_{i}(k)$ denotes the $\mathrm{y}$-coordinate of the object's prediction position, $v_{x_{i}}(k)$ and $v_{y_{i}}(k)$ denote the corresponding velocities of object, respectively $i$.

Given detection responses, we denote the set of all observations at the frame $\mathrm{k}$ as $Z=\left\{z_{j}(k)\right\}_{j=1}^{n}$, where $z_{j}(k)=\left(z_{x}^{j}(k), z_{y}^{j}(k)\right), z_{x}^{j}(k)$ and $z_{y}^{j}(k)$ denote the $\mathrm{x}-$ coordinate, y-coordinate of the observation ${ }^{j}$ respectively. The rules of the fuzzy data association approach are expressed in terms of two input variables and an output variable. The input variables $E_{i, j}(k)$ and $\Delta E_{i, j}(k)$ are defined in terms of the prediction errors and change of errors of motion and appearance models. Firstly, the normalized prediction errors of motion model is defined as follows:

$$
E_{i, j}^{M}(k)= \begin{cases}1, & \text { If }\left\|z_{j}(k)-\bar{z}_{j}(k)\right\|_{2}>\left\|z_{j}(k)-H_{k} \cdot \hat{x}_{i}(k-1)\right\|_{2} \\ \rho_{M} \cdot \frac{\left\|z_{j}(k)-\bar{z}_{j}(k)\right\|_{2}}{\left\|z_{j}(k)-H_{k} \cdot \hat{x}_{i}(k-1)\right\|_{2}}, & \text { If }\left\|z_{j}(k)-\bar{z}_{j}(k)\right\|_{2}<\left\|z_{j}(k)-H_{k} \cdot \hat{x}_{i}(k-1)\right\|_{2} \\ 0, & \text { If }\left\|z_{j}(k)-\bar{z}_{j}(k)\right\|_{2}=\left\|z_{j}(k)-H_{k} \cdot \hat{x}_{i}(k-1)\right\|_{2}\end{cases}
$$

where $\bar{z}_{j}(k)=H_{k} \cdot A_{k} \cdot \hat{\mathbf{x}}_{i}(k-1)$ denotes the predicted observation. In this paper, $H_{k}^{i}$ is an $2 \times 4$ observation transition matrix, $F_{k}^{i}$ is $4 \times 4$ state transition matrix.

Appearance is an important cue for the data association in MOT. In order to make the appearance model robust, the RGB color histogram is used to capture the statistical information of object region. To satisfy the lowcomputational cost imposed by real-time processing discrete densities, $m$-bin histograms should be used. Then, we have

Appearance model of object $i: \hat{H}\left(x_{i}\right)=\left\{H_{m}\left(x_{i}\right)\right\}_{m=1,2, \ldots, N}$
Appearance model of observation $j: \hat{H}\left(z_{j}\right)=\left\{H_{n}\left(z_{j}\right)\right\}_{n=1,2, \ldots, N}$

where $H_{m}\left(x_{i}\right)$ is the number of pixels of object $i$ in the $m$ th color bin, $H_{n}\left(z_{j}\right)$ is the number of pixels of observation $j$ in the $n$th color bin. In order to estimate the similarity between object $i$ and observation $j$, we employ the correlation coefficient method to calculate the prediction error of appearance model.

$E_{i, j}^{A}(k)=\rho_{A} \cdot \frac{\sum_{m=1}^{N}\left(H_{m}\left(x_{i}\right)-\bar{H}_{1}\right)\left(H_{m}\left(z_{i}\right)-\bar{H}_{2}\right)}{\sqrt{\sum_{m=1}^{N}\left(H_{m}\left(x_{i}\right)-\bar{H}_{1}\right)^{2} \sum_{n=1}^{N}\left(H_{n}\left(z_{i}\right)-\bar{H}_{2}\right)^{2}}}$

where

$\bar{H}_{1}=\frac{1}{N} \sum_{m=1}^{N} H_{m}\left(x_{i}\right), \quad \bar{H}_{2}=\frac{1}{N} \sum_{n=1}^{N} H_{n}\left(z_{j}\right)$

and their values lie in $[-1,1]$, Based on $E_{i, j}^{M}(k)$ and $E_{i, j}^{A}(k)$, the change errors $\Delta E_{i, j}^{M}(k)$ and $\Delta E_{i, j}^{A}(k)$ can be defined as follows:

$$
\Delta E_{i, j}^{M}(k)= \begin{cases}\frac{\Delta E_{i, j}^{M}(k)-\Delta E_{i}^{M}(k-1)}{\Delta E_{i}^{M}(k-1)}, & \text { if }\left|E_{i, j}^{M}(k)-E_{i}^{M}(k-1)\right|<\left|E_{i}^{M}(k-1)\right| \\ \frac{\Delta E_{i, j}^{M}(k)-\Delta E_{i}^{M}(k-1)}{\left|\Delta E_{i, j}^{M}(k)-\Delta E_{i}^{M}(k-1)\right|}, & \text { if }\left|E_{i, j}^{M}(k)-\Delta E_{i}^{M}(k-1)\right|>\left|E_{i}^{M}(k-1)\right| \\ 0, & \text { if }\left|E_{i, j}^{M}(k)-E_{i}^{M}(k-1)\right|=\left|E_{i}^{M}(k-1)\right|\end{cases}
$$

and

$$
\Delta E_{i, j}^{A}(k)= \begin{cases}\frac{\Delta E_{i, j}^{A}(k)-\Delta E_{i}^{A}(k-1)}{\Delta E_{i}^{A}(k-1)}, & \text { if }\left|E_{i, j}^{A}(k)-E_{i}^{A}(k-1)\right|<\left|E_{i}^{A}(k-1)\right| \\ \frac{\Delta E_{i, j}^{A}(k)-\Delta E_{i}^{A}(k-1)}{\mid \Delta E_{i, j}^{A}(k)-\Delta E_{i}^{A}(k-1)}, & \text { if }\left|E_{i, j}^{A}(k)-\Delta E_{i}^{A}(k-1)\right|>\left|E_{i}^{A}(k-1)\right| \\ 0, & \text { if }\left|E_{i, j}^{A}(k)-\Delta E_{i}^{A}(k-1)\right|=\left|E_{i}^{A}(k-1)\right|\end{cases}
$$

As a result, we define $E_{i, j}(k)$ and $\Delta E_{i, j}(k)$ to be

$E_{i, j}(k)=\sqrt{\frac{E_{i, j}^{M^{2}}(k)+E_{i, j}^{A^{2}}(k)}{3}}$

$\Delta E_{i, j}(k)=\sqrt{\frac{\Delta E_{i, j}^{M^{2}}(k)+\Delta E_{i, j}^{A^{2}}(k)}{3}}$

\subsection{Membership Functions (fuzzifier)}

The crisp values are mapped into some fuzzy sets defined in the universe of discourse of input and output. Generally, the 
more numbers of fuzzy sets we made, the more accuracy of output we got. But more numbers of fuzzy sets will increase the computation load of the algorithm. Usually, the number of fuzzy sets will be decided by experience. In the fuzzy inference system, five fuzzy sets that are labeled in the linguistic terms of zero (ZE), small positive (SP), medium positive (MP), large positive (LP), and very large positive (VP), are specified for each crisp input $\left(E_{i, j}(k)\right.$ and $\left.\Delta E_{i, j}(k)\right)$.

These membership functions of each crisp input are shown in Fig.3.

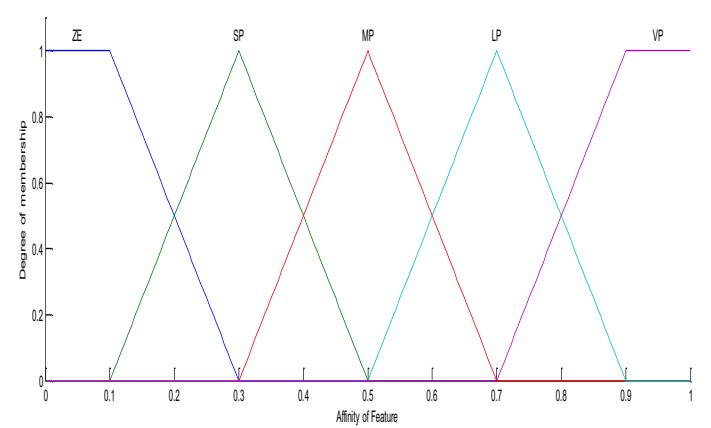

Figure. 2 Membership functions for the fuzzy sets of $E_{i, j}(k)$ and $\Delta E_{i, j}(k)$

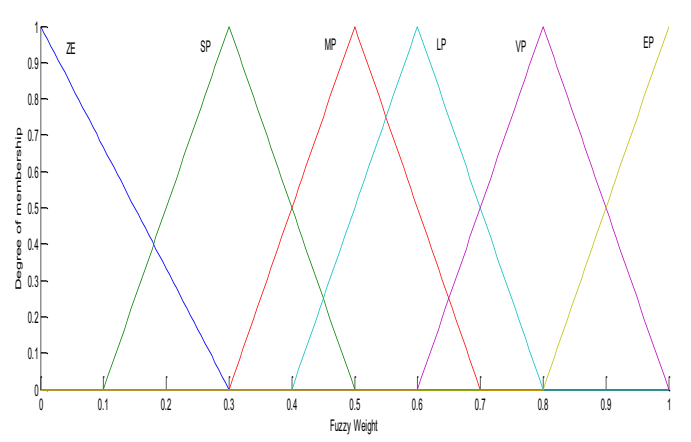

Figure.3 Membership functions for the fuzzy sets of $\mu_{i, j}(k)$

Unlike that of the input data, the output data have six fuzzy sets labeled in the linguistic terms of ZE, SP, MP, LP, VP and extremely large positive (EP). The membership functions of the fuzzy sets are defined by the triangular functions and the core of these fuzzy sets are not equally spaced. The membership functions of output are shown in Fig. 4. According to the input and output defined above, the fuzzy rules can be expressed by employing fuzzy IF-THEN rules as follows:
TABLE I. Fuzzy rule base for $\mu_{i, j}(k)$

\begin{tabular}{ccccccc}
\hline \multirow{2}{*}{$\mu_{i, j}(k)$} & \multicolumn{5}{c}{$E_{i, j}(k)$} \\
\cline { 2 - 7 } & & ZE & SP & MP & LP & VP \\
\hline \multirow{3}{*}{$\Delta E_{i, j}(k)$} & ZE & EP & VP & LP & SP & SP \\
& SP & VP & LP & MP & SP & SP \\
& MP & LP & MP & MP & SP & ZE \\
& LP & MP & MP & SP & SP & ZE \\
& VP & SP & SP & ZE & ZE & ZE \\
\hline
\end{tabular}

Tables 1 show the fuzzy rules of fuzzy inference system for the fuzzy association probability $\mu_{i, j}(k)$.The output of the weight membership functions which are used to evaluate the crisp outputs through the Max-Min compositional rule of inference technique and center of gravity (COG) defuzzification.

\subsection{0verall the Proposed Algorithm}

According to the derived results mentioned above, the structure of the proposed online visual multi-object tracking algorithm can be summarized as follows.

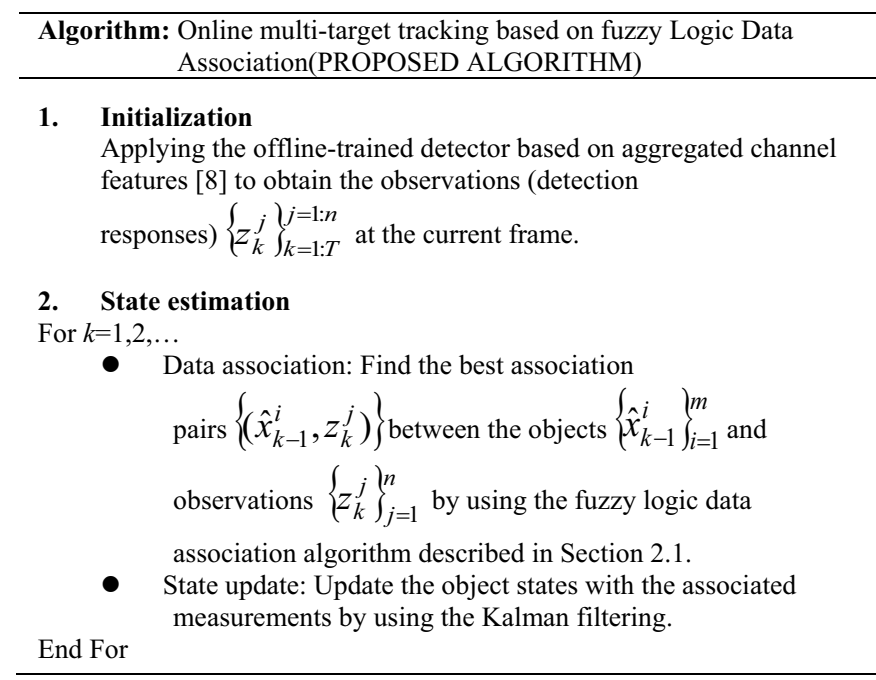

\section{Experiment Results}

We tested our algorithms on various publicly available sequences as shown in Table 2, including PETS.S2L1 dataset and PETS.S2L2 dataset.

the information of the used datasets

\begin{tabular}{rcccc}
\hline Name & FPS & $\begin{array}{c}\text { Lengt } \\
\mathrm{h}\end{array}$ & Resolution & Trajectories \\
\hline PETS.S2L1 & 7 & 795 & $768 \times 576$ & 19 \\
PETS.S2L2 & 7 & 436 & $786 \times 576$ & 42 \\
\hline
\end{tabular}

Comparisons with several state-of-the-art algorithms were made. Both qualitative analysis and quantitative evaluations were presented to show the effectiveness of our 
algorithms. The widely used CLEAR MOT metrics was employed to evaluate the proposed algorithm[7]. In order to compare the performance of the proposed algorithm with other multiple tracking algorithms, we chose two reported state-of-art trackers, such as Bae et al's proposed method[7] and $\mathrm{OM}+\mathrm{APP}[4]$.

Table 3 shows the comparison results for all metrics on all three sequences individually. Firstly, the tracking results for the PETS.S2L1 data set are compared. This data set is widely used in multi-object tracking literatures. Because the human density is low, though the dataset includes some nonlinear motion of objects and some proximity objects, all algorithms can track these objects with high tracking accuracy. Fig. 4 shows the results of the proposed algorithm. From Table 6 and Fig.4, we can see that the tracking accuracy (MOTA) of the proposed algorithm is higher than that of both [7].

Secondly, the tracking results for the PETS.S2L2 data set are compared. In this dataset, the human density is higher than the PETS.S2L1, and the objects are frequently occluded. Fig. 5 shows the results of the proposed algorithm.

\section{Conclusion}

In this paper, we proposed a new fuzzy data association approach for visual multi-object tracking. By incorporating fuzzy logic into multi-object tracking system, the association probabilities are allowed to be adjusted dynamically based on the conclusions of a set of fuzzy rules. Finally, experimental results show that the proposed algorithm provides much better performance than other state-of-art algorithms.

\section{Acknowledgement}

This work was supported by the National Natural Science Foundation of China (61301074, 61271107, and 61375015), Natural Science Foundation of the Guangdong Province of China (S2012010009417).

\section{References}

[1] Chiwoo Park, Taylor J. Woehl, James E. Evans, and Nigel D. Browning. Minimum Cost Multi-Way Data Association for Optimizing Multitarget Tracking of Interacting Objects. IEEE Transactions on Pattern Analysis and Machine Intelligence. 37 (2015)611-624.

[2] Lu Wang, Nelson Hon Ching Yung, LishengXu. Multiple-Human Tracking by Iterative Data Association and Detection Update, IEEE Transactions On Intelligent Transportation Systems. 15(2014)1886-1899.

[3] Shu Tian, Fei Yuan, Gui-Song Xia. Multi-object tracking with inter-feedback between detection and tracking, Neurocomputing, 171(2016) 768-780.

[4] A. Milan, S. Roth, and K. Schindler. Continuous energy minimization for multi-target tracking, IEEE Trans. Pattern Anal. Mach. Intell. 36(2014)58-72.

[5] B. Leibe, K. Schindler, N. Cornelis, and L. Van Gool, Coupled object detection and tracking from static cameras and moving vehicles, IEEE Trans. Pattern Anal. Mach. Intell.,30(2008)1683-1698.

[6] B. Benfold and I. Reid. Stable multi-target tracking in real-time surveillance video, in Proc. IEEE Conf. Comput. Vis. Pattern Recog., 2011, pp. 3457-3464.

[7] Seung-Hwan Bae, Kuk-Jin Yoon. Robust Online Multiobject Tracking With Data Association and Track Management,IEEE TRANSACTIONS ON IMAGE PROCESSING, 33(2014)2820-2833.

[8] P. Dollár, R. Appel, S. Belongie, et al. Fast feature pyramids for object detection. IEEE Trans. Pattern Anal. Mach. Intell., 36(8)(2014)1532-1545.

Performance Comparison of Tracking Results on Two Different Datasets

\begin{tabular}{|c|c|c|c|c|c|c|c|}
\hline Sequence & Method & MOTA & MOTP & IDS & MT & ML & $\mathrm{FG}$ \\
\hline \multirow{3}{*}{ PETS.S2L1 } & Bae et al.[7] & 83.0 & 69.6 & 4 & 100 & 0 & 4 \\
\hline & $\mathrm{OM}+\mathrm{APP}[4]$ & 90.6 & 80.2 & 11 & 21 & 1 & 6 \\
\hline & Proposed Algorithm & 86.2 & 65.4 & 11 & 15 & 0 & 58 \\
\hline \multirow{3}{*}{ PETS.S2L2 } & Bae et al.[7] & 30.2 & 69.2 & 284 & 2.4 & 19.0 & 499 \\
\hline & $\mathrm{OM}+\mathrm{APP}[4]$ & 56.9 & 59.4 & 99 & 28 & 12 & 73 \\
\hline & Proposed Algorithm & 51.5 & 66.1 & 154 & 11 & 3 & 362 \\
\hline
\end{tabular}



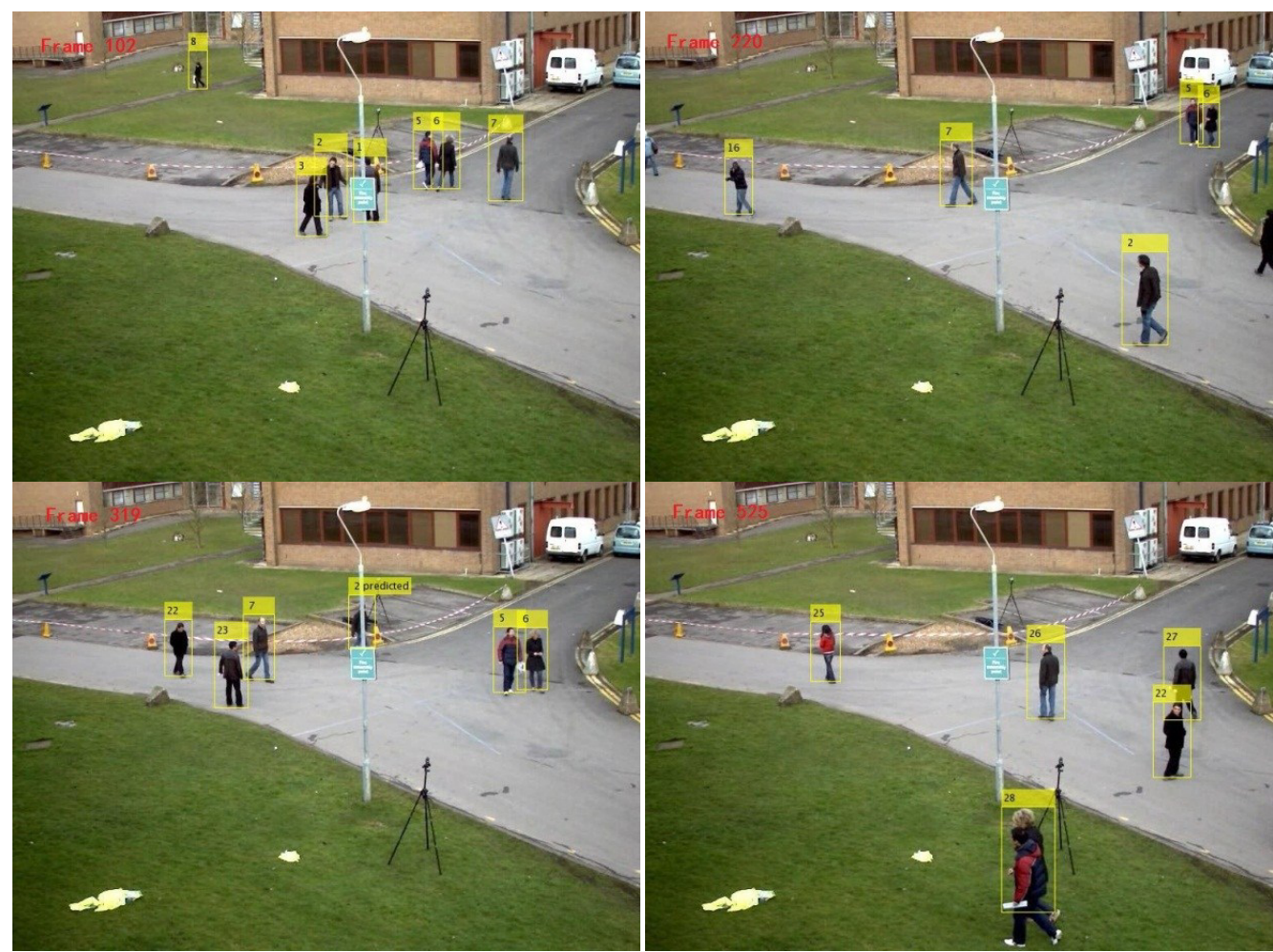

Figure.4. Results of the proposed algorithm on PETS.S2L1 dataset
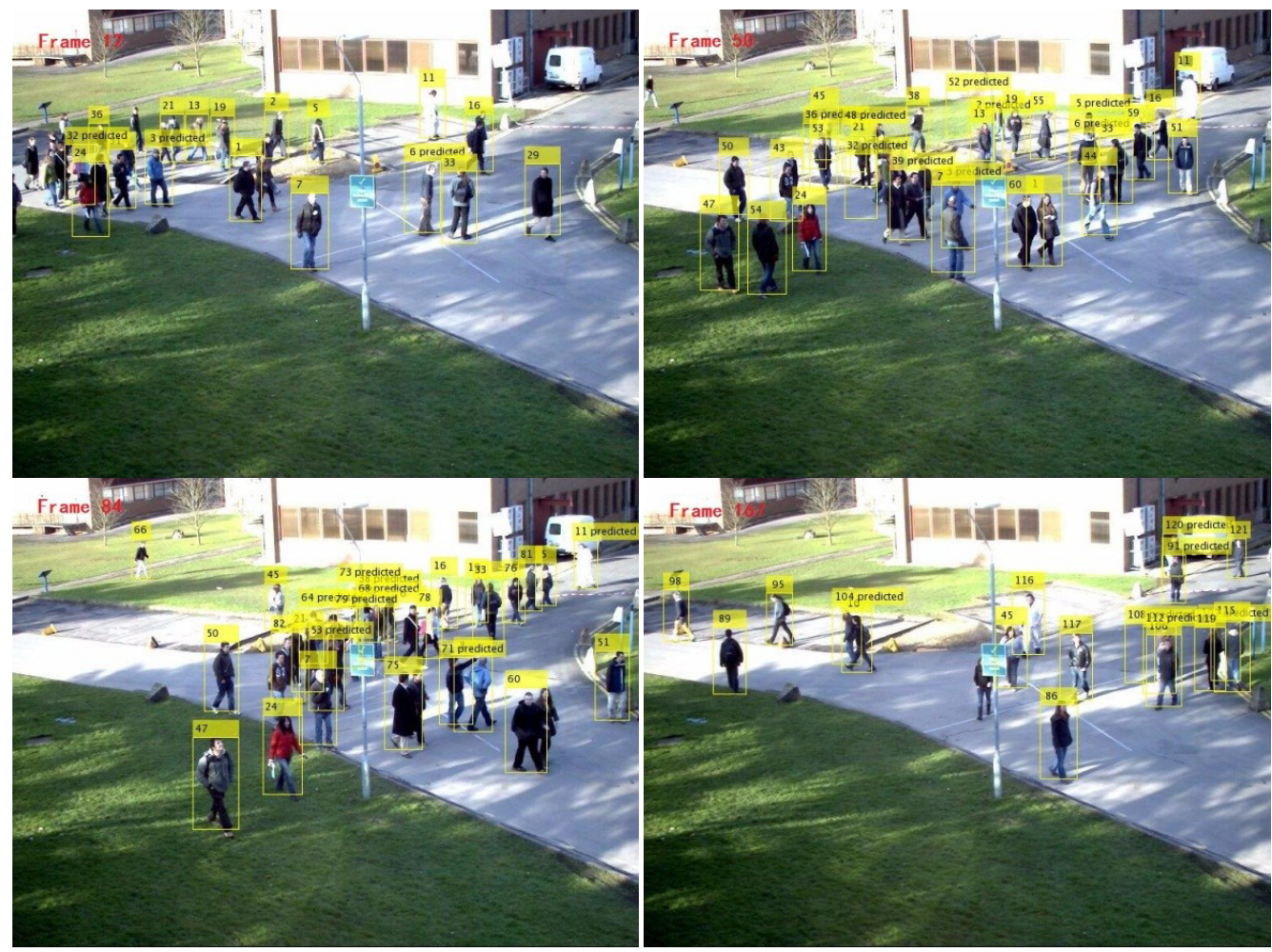

Figure.5. Results of the proposed algorithm on PETS.S2L2 dataset 